\title{
The Serum Level of IL-1B Correlates with the Activity of Chronic Pulmonary Aspergillosis
}

\author{
Mengling Zhan, ${ }^{1}$ Benyong Xu, ${ }^{1}$ Lan Zhao, ${ }^{2}$ Bing $\mathrm{Li}^{2}{ }^{2}$ Liyun Xu, ${ }^{2}$ Qiuhong Sun, ${ }^{2}$ Jun Zhang, \\ Zhemin Zhang $\mathbb{D}^{2}$, and Haiqing Chu $\mathbb{D}^{2}$ \\ ${ }^{1}$ Tongji University School of Medicine, Shanghai 200092, China \\ ${ }^{2}$ Department of Respiratory Medicine, Shanghai Pulmonary Hospital, Tongji University School of Medicine, \\ Shanghai 200433, China \\ ${ }^{3}$ Division of Hematology, Oncology and Blood \& Marrow Transplantation, Department of Internal Medicine, \\ Holden Comprehensive Cancer Center, University of Iowa Carver College of Medicine, Iowa City, IA 52242, USA \\ Correspondence should be addressed to Zhemin Zhang; zhemindoc@163.com and Haiqing Chu; chu_haiqing@126.com
}

Received 26 May 2018; Revised 26 August 2018; Accepted 2 September 2018; Published 27 September 2018

Academic Editor: Alice M. Turner

Copyright ( $\odot 2018$ Mengling Zhan et al. This is an open access article distributed under the Creative Commons Attribution License, which permits unrestricted use, distribution, and reproduction in any medium, provided the original work is properly cited.

\begin{abstract}
Background. Until now, there have been no objective criteria to determine the activity of chronic pulmonary aspergillosis (CPA). This study aims to analyze the correlation between serum level of IL-1B and the activity of CPA and to determine whether serum IL-1B could be used to assess the activity of CPA. Methods. A total of 469 newly diagnosed CPA patients were enrolled. Correlation analysis in the whole subjects showed that only IL-1B level was associated with the activity of CPA. Then, 381 cases with factors significantly affecting IL-1B expression was excluded through multiple linear regression; the remaining 88 patients were divided into high IL-1B group and low IL-1B group, according to the median value of serum IL-1B, for subgroup analysis. A retrospective comparative analysis was subsequently performed between the two groups, including the clinical manifestation, microbiology and laboratory tests results, and imaging findings. We further investigated the relationship between IL-1B levels and CT characteristic which acted as the indicator of CPA activity, as well as changes in IL-1B level before and after surgery. Results. For all patients, correlation analysis revealed that IL-1B level correlated with both cavitary diameter $(P=0.035)$ and aspergilloma size $(P<0.047)$ but not with the thickness of the cavity $(P=0.479)$. In subgroup comparative analysis, CT characteristics suggested that high activity of $\mathrm{CPA}$, such as cavitary $(27 / 44$ vs $13 / 44, P=0.003)$ and aspergilloma lesions $(25 / 44$ vs. $11 / 44, P<0.002)$, were more frequently found in high IL-1B group. The cavity diameter $(P<0.001)$, aspergilloma size $(P=0.006)$, and cavity wall thickness $(P=0.023)$ were significantly different between the two groups. When Spearman correlation analysis was performed once again in subgroup, an even stronger relationship of serum IL-1B with the cavity diameter $(R s=0.501, P=0.002)$ and aspergilloma size ( $R s=0.615, P=0.001)$ was observed. Interestingly, a significant reduction of IL-1B level was observed after successful resection of CPA lesions. Conclusion. Higher level of serum IL-1B is associated with more severe cavitary and aspergilloma lesions, which are indicative of more active CPA. In addition, IL-1B level reduced accordingly after lesion resection. Measuring IL-1B level therefore could be served as a convenient method to monitor the activity of CPA and be a potential predictive/prognostic marker for treatment response.
\end{abstract}

\section{Introduction}

Chronic pulmonary aspergillosis (CPA) is a chronic form of debilitating aspergillus infection that affects patients with pre-existing structural lung diseases [1]. It was reported that CPA affected 240,000 people in Europe and caused tremendous global burden following pulmonary tuberculosis
(TB) [2-4]. CPA may also result in significant mortality, with a 5 -year survival rate of $17.5 \%-62 \%$ without surgical resection and 15\%-20\% without any intervention [5-7].

The relationship between cytokines and fungal infections has been gaining interest among clinicians and scientists. Various studies on cytokine gene single-nucleotide polymorphisms (SNP), expression level, and related pathways in 
fungal infections have demonstrated that distinct cytokine profiles are closely related to inflammasome activation and disease activity, some of which suggested that cytokines may correlate with the activity of CPA [8-11]. For example, several papers reported that interleukin (IL)-1, IL-15, and interferon- $\gamma$ could contribute to the pathogenesis in chronic cavitary pulmonary aspergillosis (CCPA) [8-10] and reduced CD40L and sCD40L, as well as increased IL-10 may compromise the immune response against aspergillus in patients with chronic necrotizing pulmonary aspergillosis (CNPA) [11].

IL-1 has been considered as a crucial cytokine for hosts to defend against a broad range of pathogens. The classical IL-1 cytokines are IL-1A and IL-1B [12]. Over the past years, the IL-1 cytokines, particular IL-1B, have been studied for their role in the antifungal host response against Aspergillus fumigatus infection. IL-1B is primarily produced by innate immune cells such as monocytes, macrophages, and dendritic cells upon activation. It is a potent proinflammatory cytokine that is tightly regulated by immune cells. Previous studies have demonstrated that active and growing fungi, such as swollen conidia, germinated conidia [13], hyphal fragments [14], and live conidia [15], were more potent in inducing IL-1B expression, and persistently elevated IL-1B could be a sign of strong fungal activity as well as on-going inflammation induced by host immune response to active fungus. However, evidence supporting a direct link of IL-1B with CPA is still lacking. Thus, we hypothesize that IL-1B also plays an import role in chronic pulmonary aspergillosis.

In the present study, we have endeavored to show that only serum IL-1B, among several proinflammatory cytokines and other traditional measures of inflammation, acted as a promising disease biomarker in predicting the activity of $\mathrm{CPA}$, by exploring its association with the patients' clinical manifestations, microbiological manifestations, laboratory test results, and imaging findings.

\section{Patients and Methods}

2.1. Patient Selection. A total of 488 newly diagnosed CPA patients were recruited, among which, 19 subjects were excluded due to lack of complete image and blood inflammatory cytokine data. Thus, finally 469 subjects participated in the current study (Table 1). Patients were initially diagnosed and treated in Shanghai Pulmonary Hospital, Tongji University, from January 2009 to December 2015. The diagnostic criteria were in accordance with the European Society for Clinical Microbiology and Infectious Diseases (ESCMID) and the European Respiratory Society (ERS) Guideline of 2016 [16]. All the patients were documented well with detailed clinical and radiographic information. Microbiological and laboratory results of sputum, bronchial lavage fluid, and blood were collected before initial diagnosis. The multiple linear regression model for predicting serum IL-1B (using patient baseline characteristics, treatment status, and associated diseases as covariates in all the subjects with CPA) was conducted to screen for factors influencing serum IL-1B (Table 2). A total of 381 cases with any of these factors were excluded. The remaining
88 patients with CPA were divided into the high and low IL$1 \mathrm{~B}$ groups according to the median serum IL-1B concentration of $20.3 \mathrm{ng} / \mathrm{L}$ for further subgroup analysis (Table 1). Among these patients, 22 received surgical treatment, and changes in serum IL-1B were evaluated. The study was approved by the Ethical Committee of Tongji University, Shanghai Pulmonary Hospital. All the participants signed informed consent for any procedures that are relevant to this study.

2.2. CT Scanning. All CT images were obtained from the Radiology Department of Shanghai Pulmonary Hospital. CT scanning was performed within 1 week of CPA diagnosis. Multislice spiral CT (Philips Brilliance 64) was used for routine CT scanning with $5 \mathrm{~mm}$ thick images at $5 \mathrm{~mm}$ intervals. High-resolution computed tomography (HRCT) images were obtained by scanning using $1 \mathrm{~mm}$ thickness at $10 \mathrm{~mm}$ intervals. The scanning range was from the lung apex to the costophrenic angle under the maximum inhalation. CT images were analyzed and interpreted by two radiologists and a pulmonologist who were blinded for the microbiological test results. All images were transmitted to the PACS system, and after multislice recombination, the threedimensional image was reconstructed and then measured. The diameter of the Aspergilloma was measured in each layer, and direction and the maximum value were taken. The cavity diameter was defined as the average of the anteroposterior, transverse, and axial diameter measured in the mediastinal window. The thickness of the cavity wall was gauged in the front, rear, left, and right wall, and the average was taken. The largest lesion was measured when the lesion was multiple. The final interpretation was established by the consensus achieved among those three experts. The radiographic evaluation was based on Agarwal et al. and Godet et al.'s study $[17,18]$.

2.3. Blood Inflammatory Cytokines Test. All patients had fasted for more than 12 hours before $5 \mathrm{ml}$ of peripheral venous blood was extracted in the next morning. The blood test was done around the time when the CT scan was performed. For the postoperative blood test, the blood samples were collected 3 months after surgery. All collected peripheral blood samples were centrifuged for $15 \mathrm{~min}\left(4^{\circ} \mathrm{C}\right.$; $3000 \mathrm{r} / \mathrm{min}$ ), and the sera were collected for determination the level of inflammatory cytokines including IL-1B, IL-2, IL-6, IL-5, tumor necrosis factor- (TNF-) $\alpha$ and interferon- $\gamma$. Enzyme-linked immunosorbent assay (ELISA) was used, and the ELISA kit was purchased from BioLegend, Inc (US).

2.4. Statistical Analysis. All statistical analyses were conducted using SPSS20.0 (IBM, Armonk, NY, USA). The data were compared using Student's $t$-test (normally distributed data) or Mann-Whitney $U$ test (nonnormally distributed data) for continuous variables, and Pearson $\chi^{2}$ test or Fisher's exact test for categorical variables. Student's paired $t$-test was performed to compare the pre- and postoperative serum IL-1B levels in selected CPA patients. Correlations 
TABLE 1: Clinical characteristics of subjects with CPA in the whole and subgroup analysis.

\begin{tabular}{|c|c|c|c|c|c|}
\hline & \multirow[b]{2}{*}{$\begin{array}{l}\text { Whole group } \\
\quad(n=469)\end{array}$} & \multirow[b]{2}{*}{$\begin{array}{c}\text { Total } \\
(n=88)\end{array}$} & \multicolumn{2}{|c|}{ Subgroup } & \multirow[b]{2}{*}{$\begin{array}{c}P \\
\text { value }\end{array}$} \\
\hline & & & $\begin{array}{c}\text { Serum } \\
\text { IL-1B } \geq 20.3 \mathrm{ng} / \mathrm{L} \\
(n=44)\end{array}$ & $\begin{array}{c}\text { Serum } \\
\text { IL-1B }<20.3 \mathrm{ng} / \mathrm{L} \\
(n=44)\end{array}$ & \\
\hline Age, years & $57(48.0,66.0)$ & $55(42.0,62.0)$ & $56.5(46.0,62.0)$ & $50.5(40.3,63.0)$ & 0.356 \\
\hline Gender, male & $279(59.5)$ & $52(59.1)$ & $24(54.5)$ & $28(63.6)$ & 0.386 \\
\hline BMI, $\mathrm{kg} / \mathrm{m}^{2}$ & $21.1 \pm 3.6$ & $21.4 \pm 3.9$ & $21.6 \pm 4.1$ & $21.3 \pm 3.8$ & 0.729 \\
\hline $\begin{array}{l}\text { Smoking history, yes, } \\
\text { pulmonary underlying } \\
\text { diseases }\end{array}$ & $144(30.7)$ & $26(29.5)$ & $11(25.0)$ & $15(34.1)$ & 0.350 \\
\hline & $180(38.4)$ & $40(45.5)$ & $22(50.0)$ & $18(40.9)$ & 0.392 \\
\hline & $87(18.6)$ & - & - & - & - \\
\hline NTM infection & $30(6.4)$ & - & - & - & - \\
\hline $\begin{array}{l}\text { Other acute } \\
\text { respiratory } \\
\text { infections }\end{array}$ & $59(12.6)$ & - & - & - & - \\
\hline Lung abscess & $12(2.6)$ & - & - & - & - \\
\hline Cancers & $60(12.8)$ & - & - & - & - \\
\hline \multirow{2}{*}{ COPD } & $212(45.2)$ & $47(53.4)$ & $24(54.5)$ & $23(52.3)$ & 0.831 \\
\hline & $53(11.3)$ & - & - & - & - \\
\hline Sarcoidosis & $34(7.3)$ & $5(5.7)$ & $2(4.5)$ & $3(6.8)$ & 1.000 \\
\hline AIP & $12(2.6)$ & - & - & - & - \\
\hline Other ILD & $53(11.3)$ & $4(4.8)$ & $3(6.8)$ & $1(2.3)$ & 0.616 \\
\hline \multicolumn{6}{|l|}{ Systemic comorbidities } \\
\hline $\mathrm{DM}$ & $53(11.3)$ & $7(8.0)$ & $3(6.8)$ & $4(9.1)$ & 1.000 \\
\hline Hypertension & $60(12.8)$ & $14(15.9)$ & $6(13.6)$ & $8(18.2)$ & 0.560 \\
\hline Cardiac diseases & $60(12.8)$ & $9(10.2)$ & $3(6.8)$ & $6(13.6)$ & 0.484 \\
\hline $\begin{array}{l}\text { Cerebrovascular } \\
\text { diseases }\end{array}$ & $14(2.98)$ & $2(2.3)$ & $0(0)$ & $2(4.5)$ & 0.494 \\
\hline Systemic CS therapy & $36(7.7)$ & - & - & - & - \\
\hline Systemic IS therapy & $32(6.8)$ & - & - & - & - \\
\hline $\begin{array}{l}\text { Pulmonary surgery } \\
\text { history }\end{array}$ & $38(8.1)$ & $9(10.2)$ & $2(4.5)$ & $7(15.9)$ & 0.157 \\
\hline Symptoms & & & & & \\
\hline Cough & $460(98.1)$ & $85(96.6)$ & $44(100)$ & $41(93.2)$ & 0.241 \\
\hline Sputum production & $455(97.0)$ & $82(93.2)$ & $42(95.5)$ & $40(90.9)$ & 0.676 \\
\hline Hemosputum & $272(58.0)$ & $45(51.1)$ & $26(59.1)$ & $19(43.2)$ & 0.135 \\
\hline Fever & $158(33.7)$ & $19(21.6)$ & $8(18.2)$ & $11(25.0)$ & 0.437 \\
\hline Chest pain & $58(12.36)$ & $5(5.68)$ & $2(4.5)$ & $3(6.8)$ & 1.000 \\
\hline Fatigue & $79(16.8)$ & $4(4.5)$ & $3(6.8)$ & $1(2.3)$ & 0.616 \\
\hline Radiological features & & & & & \\
\hline Cavity & $235(50.1)$ & $40(45.5)$ & $27(61.4)$ & $13(29.5)$ & 0.003 \\
\hline Aspergilloma & $212(45.2)$ & $36(40.9)$ & $25(56.8)$ & $11(25.0)$ & 0.002 \\
\hline Bronchiectasis & $408(87.0)$ & $83(94.3)$ & $23(52.3)$ & $23(52.3)$ & 1.000 \\
\hline Patch & $451(96.2)$ & $82(93.2)$ & $43(97.7)$ & $39(88.6)$ & 0.091 \\
\hline Consolidation & $363(77.4)$ & $60(68.2)$ & $27(61.4)$ & $33(75.0)$ & 0.170 \\
\hline Nodules & $332(70.8)$ & $58(65.9)$ & $31(70.5)$ & $27(61.4)$ & 0.368 \\
\hline Tree-in-bud pattern & $85(18.1)$ & $8(9.1)$ & $3(6.8)$ & $5(11.4)$ & 0.713 \\
\hline Atelectasis & $28(6.0)$ & $3(3.40)$ & $0(0)$ & $3(6.8)$ & 0.241 \\
\hline Pleural effusion & $79(16.8)$ & $8(9.1)$ & $5(11.4)$ & $3(6.8)$ & 0.713 \\
\hline Pleural thickening & $192(40.9)$ & $39(44.3)$ & $22(50)$ & $17(38.6)$ & 0.283 \\
\hline Interstitial fibrosis & $53(11.3)$ & $4(4.8)$ & $2(4.5)$ & $2(4.5)$ & 1.000 \\
\hline $\begin{array}{l}\text { Lung volume } \\
\text { reduction }\end{array}$ & $47(10.0)$ & $6(6.8)$ & $2(4.5)$ & $4(9.1)$ & 0.676 \\
\hline GGO & $53(11.3)$ & $8(9.1)$ & $2(4.5)$ & $6(13.6)$ & 0.266 \\
\hline $\begin{array}{l}\text { Cavity diameter } \\
(\mathrm{cm})\end{array}$ & $3.6(2.6,4.8)$ & $3.4(2.1,4.8)$ & $4.6(2.8,5.0)$ & $2.0(1.6,2.7)$ & 0.000 \\
\hline $\begin{array}{l}\text { Aspergilloma size } \\
(\mathrm{cm})\end{array}$ & $2.4(1.7,3.3)$ & $1.8(1.5,3.5)$ & $2.5(1.6,3.8)$ & $1.5(0.9,1.9)$ & 0.006 \\
\hline $\begin{array}{l}\text { Cavity wall } \\
\text { thickness }(\mathrm{cm})\end{array}$ & $0.7(0.5,1.0)$ & $0.7(0.3,1.1)$ & $0.9(0.4,1.2)$ & $0.4(0.2,0.7)$ & 0.023 \\
\hline
\end{tabular}


TABle 1: Continued.

\begin{tabular}{|c|c|c|c|c|c|}
\hline & & & & & \\
\hline & $\begin{array}{l}\text { Whole group } \\
\quad(n=469)\end{array}$ & $\begin{array}{c}\text { Total } \\
(n=88)\end{array}$ & $\begin{array}{c}\text { Serum } \\
\text { IL-1B } \geq 20.3 \mathrm{ng} / \mathrm{L} \\
(n=44)\end{array}$ & $\begin{array}{c}\text { Serum } \\
\text { IL-1B }<20.3 \mathrm{ng} / \mathrm{L} \\
(n=44) \\
\end{array}$ & $\begin{array}{c}P \\
\text { value }\end{array}$ \\
\hline G test, positive & $95(20.3)$ & $16(18.2)$ & $9(20.5)$ & $7(15.9)$ & 0.580 \\
\hline GM test, positive & & & & & \\
\hline Blood & $211(45.0)$ & $43(48.9)$ & $25(56.8)$ & $18(40.9)$ & 0.135 \\
\hline BWF & $338(72.1)$ & $60(68.2)$ & $32(72.7)$ & $28(63.6)$ & 0.360 \\
\hline $\begin{array}{l}\text { Aspergillus antibody, } \\
\text { positive }\end{array}$ & $276(58.8)$ & $55(62.5)$ & $25(56.8)$ & $30(68.2)$ & 0.271 \\
\hline Culture proof & & & & & \\
\hline Sputum & $177(37.7)$ & $32(36.4)$ & $17(38.6)$ & $15(34.1)$ & 0.658 \\
\hline BWF & $165(35.2)$ & $32(36.4)$ & $19(43.2)$ & $13(29.5)$ & 0.184 \\
\hline Laboratory test result & & & & & \\
\hline ALB & $39.1 \pm 5.7$ & $38.9 \pm 5.6$ & $38.6 \pm 4.9$ & $39.1 \pm 6.2$ & 0.699 \\
\hline WBC & $7.2(5.4,9.3)$ & $7.5(5.3,9.0)$ & $7.1(5.1,9.6)$ & $8.0(5.4,8.8)$ & 0.867 \\
\hline CRP & $6.9(2.7,20.4)$ & $5.9(2.1,18.8)$ & $5.8(2.0,26.2)$ & $6.0(2.4,8.3)$ & 0.987 \\
\hline ESR & $33.3(16.4,51.3)$ & $26.9(11.8,51.6)$ & $26.3(11.5,50.0)$ & $30.7(12.2,52.7)$ & 0.739 \\
\hline Inflammatory cytokin & & & & & \\
\hline IL-1B & $26.1(15.2,50.6)$ & $20.3(15.2,42.3)$ & $42.2(25.2,57.6)$ & $15.2(11.7,17.3)$ & 0.000 \\
\hline IL-2 & $144.9(102.2,193.1)$ & $110.9(75.1,159.8)$ & $112.5(76.3,161.1)$ & $101.0(69.9,147.3)$ & 0.413 \\
\hline IL-5 & $39.2(26.8,62.3)$ & $37.7(26.4,61.5)$ & $46.8(27.0,62.4)$ & $32.8(25.1,50.1)$ & 0.090 \\
\hline IL-6 & $45.6(23.0,94.8)$ & $39.8(26.7,73.9)$ & $56.0(27.6,77.2)$ & $33.9(24.6,66.6)$ & 0.123 \\
\hline TNF- $\alpha$ & $52.3(30.3,96.1)$ & $53.6(33.7,90.0)$ & $65.4(33.5,96.0)$ & $46.5(33.9,79.4)$ & 0.174 \\
\hline$\gamma$-IFN & $16.6(11.6,27.5)$ & $17.8(11.8,27.0)$ & $20.0(11.9,31.4)$ & $16.8(11.7,22.3)$ & 0.129 \\
\hline
\end{tabular}

Data are presented as mean \pm standard deviation or median (interquartile range) or number (percentage). AIP: acute interstitial pneumonia; ALB: albumin; BMI: body mass index; BWF: bronchial washing fluid; COPD: chronic obstructive pulmonary disease; CRP: C-reactive protein; CS: corticosteroid; DM: diabetes mellitus; ESR: erythrocyte sedimentation rate; GGO: ground glass opacity; IFN: interferon; IL: interleukin; ILD: interstitial lung disease; IS: immunosuppressive; NTM: nontuberculous mycobacterium; TB: tuberculosis; TNF: tumor necrosis factor; WBC: white blood cell.

were examined with Spearman's rank test. Serum IL-1B levels were analyzed by multiple stepwise regression until the most parsimonious model was achieved. Univariate $P$ values of 0.2 entered the multivariate model. $P$ values of $<0.05$ were considered statistically significant.

\section{Results}

3.1. Patient Characteristics. Eligible patients' characteristics, CT findings, and related test results for all patients and patients in subgroup are shown in Table 1. Based on the median value $(20.3 \mathrm{ng} / \mathrm{L}$, ranged from 0 to $499 \mathrm{ng} / \mathrm{L}), 44$ cases were classed in high IL-1B group and 44 in low IL-1B group. There was no difference in basic characteristics, common laboratory test, aspergillus antibody, and GM test results between the two groups. Other commonly used cytokines such as IL-2, IL-6, IL-5, TNF- $\alpha$, and interferon- $\gamma$ revealed no significant difference as well (Table 1).

3.2. Relationship between Serum IL-1B Level and Radiographic Findings. For all patients, Spearman's rank correlation analysis revealed a mild but statistically significant correlation of IL-1B level with both cavitary diameter $(P=0.035)$ and aspergilloma size $(P=0.047)$, but not with the thickness of the cavity $(P=0.479)$ (Table 3$)$. In view of numerous accompanying factors and comorbidities potentially affecting the immunological status in our recruited subjects, we constructed a multiple linear regression model for predicting serum IL-1B using age, gender, BMI, smoking status, TB, NTM infection, lung abscess, other acute respiratory infection, cancer, COPD, AIP, sarcoidosis, other interstitial diseases, diabetes mellitus, hypertension, cardiac disease, cerebrovascular disease, systemic CS or IS therapy, and pulmonary surgery history as covariates in all the subjects with chronic pulmonary aspergillosis to screen for factors influencing serum IL-1B. The results indicated that active tuberculosis infection, NTM infection, lung abscess, other acute respiratory infections, cancer, AECOPD, AIP, and sarcoidosis positively correlated with serum IL-1B, while systemic CS or IS therapy led to a suppression in IL-1B expression. Accordingly, a total of 381 cases with any of these factors were excluded, and a subgroup analysis was performed in the remaining 88 subjects. When Spearman's correlation analysis was performed once again, and an even stronger relationship of serum IL-1B with the cavity diameter $(R s=0.501, P=0.002)$ and aspergilloma size ( $R s=0.615, P=0.001$ ) was observed (Figure 1). Besides, CT characteristics suggested that high activity of CPA, such as cavitary $(27 / 44$ vs $13 / 44, P=0.003)$ and aspergilloma lesions (25/44 vs. $11 / 44, P=0.002)$, were more frequently found in high IL-1B group. The cavity diameter (median, $4.6 \mathrm{~cm}$ 
TABLE 2: Multiple linear regression model for predicting serum IL-1B using age, gender, BMI, smoking status, TB, NTM infection, lung abscess, other acute respiratory infections, cancer, COPD, AIP, sarcoidosis, other interstitial diseases, diabetes mellitus, hypertension, cardiac disease, cerebrovascular disease, systemic CS or IS therapy, and pulmonary surgery history as covariates in all the subjects with chronic pulmonary aspergillosis.

\begin{tabular}{|c|c|c|c|c|}
\hline \multicolumn{2}{|c|}{ Comorbid conditions } & $\beta$ Coefficient & Standard error & $P$ value \\
\hline & None (reference) & - & - & - \\
\hline \multirow[t]{2}{*}{$\mathrm{TB}$} & Treated & 0.003 & 0.106 & 0.978 \\
\hline & Active/being treated & 0.475 & 0.138 & 0.001 \\
\hline \multicolumn{2}{|c|}{ NTM infection } & 0.538 & 0.196 & 0.006 \\
\hline \multicolumn{2}{|c|}{ Lung abscess } & 0.975 & 0.304 & 0.001 \\
\hline \multicolumn{2}{|c|}{ Other acute respiratory infection } & 0.547 & 0.151 & 0.000 \\
\hline \multicolumn{2}{|c|}{ Cancer } & 0.542 & 0.147 & 0.000 \\
\hline & None (reference) & - & - & - \\
\hline \multirow[t]{2}{*}{ COPD } & Stable & 0.119 & 0.102 & 0.247 \\
\hline & Acute & 0.612 & 0.164 & 0.000 \\
\hline \multicolumn{2}{|l|}{ AIP } & 0.818 & 0.305 & 0.008 \\
\hline \multicolumn{2}{|c|}{ Sarcoidosis } & 0.407 & 0.185 & 0.028 \\
\hline \multicolumn{2}{|c|}{ Systemic CS or IS therapy } & -0.297 & 0.144 & 0.039 \\
\hline
\end{tabular}

AIP: acute interstitial pneumonia; COPD: chronic obstructive pulmonary disease; CS: corticosteroid; ILD: interstitial lung disease; IS: immunosuppressive; NTM: nontuberculous mycobacterium.

TABle 3: Correlation of serum inflammatory factors with the activity of chronic pulmonary aspergillosis in the whole subjects.

\begin{tabular}{lccccc}
\hline & \multicolumn{2}{c}{ Cavity diameter } & \multicolumn{2}{c}{ Aspergilloma size } & \multicolumn{2}{c}{ Cavity wall thickness } \\
& Coefficient & $P$ value & Coefficient & $P$ value & $P$ Coefficient \\
\hline WBC & 0.088 & 0.149 & 0.034 & 0.587 & 0.065 \\
ESR & 0.075 & 0.215 & 0.097 & 0.118 & 0.098 \\
CRP & 0.113 & 0.064 & 0.078 & 0.210 & 0.103 \\
IL-1B & 0.128 & 0.035 & 0.123 & 0.047 & 0.043 \\
IL-2 & 0.073 & 0.229 & 0.014 & 0.827 & 0.094 \\
IL-5 & 0.053 & 0.387 & 0.098 & 0.116 & 0.085 \\
IL-6 & 0.091 & 0.135 & 0.079 & 0.207 & 0.089 \\
TNF- $\alpha$ & 0.083 & 0.174 & 0.049 & 0.435 & 0.109 \\
$\gamma$-IFN & 0.063 & 0.300 & 0.060 & 0.337 & 0.094 \\
\hline
\end{tabular}

CRP: C-reactive protein; ESR: erythrocyte sedimentation rate; IFN: interferon; IL: interleukin; TNF: tumor necrosis factor; WBC: white blood cell.

(IQR, 2.8 to $5.0 \mathrm{~cm}$ ) vs. $2.0 \mathrm{~cm}$ (IQR, 1.6 to $2.7 \mathrm{~cm}$ ); $P=0.001)$, aspergilloma size (median, $2.5 \mathrm{~cm}(\mathrm{IQR}, 1.6$ to $3.8 \mathrm{~cm}$ ) vs. $1.5 \mathrm{~cm}$ (IQR, 0.9 to $1.9 \mathrm{~cm}) ; P=0.006)$, and cavity wall thickness (median, $0.9 \mathrm{~cm}$ (IQR, 0.4 to $1.2 \mathrm{~cm}$ ) vs. $0.4 \mathrm{~cm}$ (IQR, 0.2 to $0.7 \mathrm{~cm}$ ); $P=0.023$ ) were significantly different between the two groups.

3.3. Changes in Serum $I L-1 B$ before and after Surgery. Figure 2 shows the cavity size and the change of serum IL-1B level in 22 patients who got their cavitary lesions surgically resected. There was a clear upward trend of IL-1B with the increase in lesion size before the operation. Three months after surgery, we observed almost universal reduction of serum IL-1B levels $(47.4 \pm 42.7 \mathrm{ng} / \mathrm{L}$ vs $23.7 \pm 9.4 \mathrm{ng} / \mathrm{L}$, $P=0.001$ ) (Figure 3), with most prominent change among those whose baseline IL-1B level was more than 20 ng/L (Figure 2).

\section{Discussion}

To our knowledge, this is the first study exploring correlation between IL-1B level and clinical manifestations in patients with CPA. In our study, CPA patients' underlying disease (mainly TB and COPD), constitutional symptoms (weight loss, productive cough, and hemoptysis), routine laboratory test measures (ESR, CRP, etc.), aspergillus antibody titer, and GM test results were similar to those of reported studies [19]. Moreover, we observed higher serum IL-1B levels in $\mathrm{CPA}$ were associated with more and bigger cavitary and aspergilloma lesions, a finding, which were corroborated by a robust reduction in serum IL-1B level after surgical removal of the CPA cavitary lesions. However, this association was substantially confounded by a couple of comorbid conditions and accompanying medications. For example, AECOPD and active TB infection upregulated, while systemic CS or IS therapy suppressed the expression of IL-1B, and thus should be on guard in the clinical use of this cytokine as a monitor of the activity of CPA. The lack in the association between serum IL-1B and cavity wall thickness suggests factors other than IL-1B or inflammatory cytokines play a role.

Although previous studies suggested the associations between $\mathrm{CPA}$ and various inflammatory cytokines, e.g., TNF, Tumor growth factor- (TGF-) $\beta 1$, IL-15, and IL-10 [20-23], the results are not consistent and some are difficult 


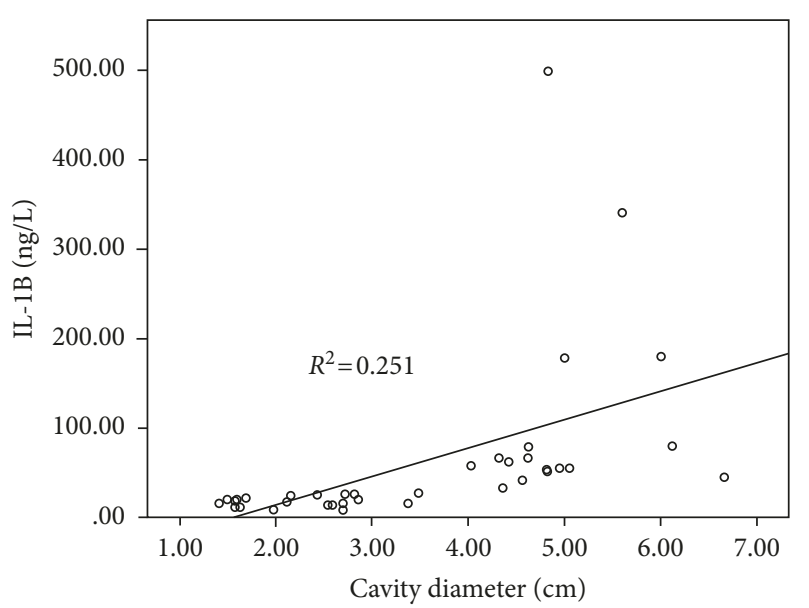

(a)

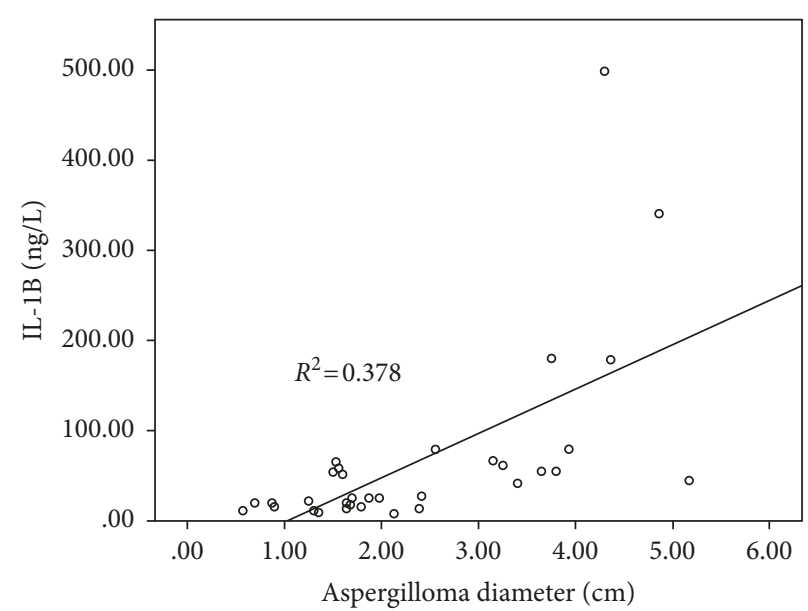

(b)

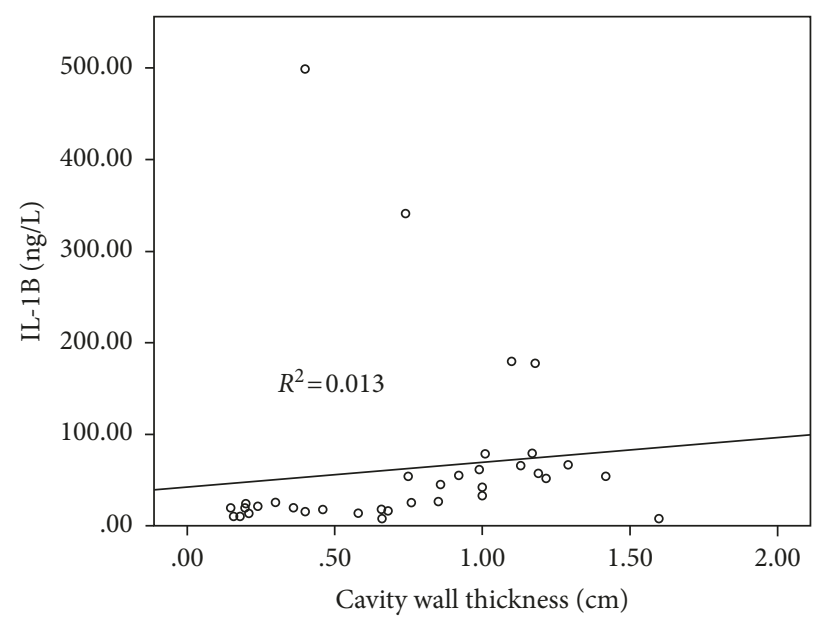

(c)

FIGURE 1: Trend analysis and correlation analysis found that IL-1B was correlated with the cavity diameter $(R s=0.501, P=0.002)(\mathrm{a})$ and aspergilloma size $(R s=0.615, P=0.001)(b)$, but not with the cavity wall thickness $(R s=0.114, P=0.496)$ (c).

to replicate. In 2014, through a comprehensive study containing clinical samples and animal models, Smith et al. revealed a remarkable relationship between IL-1 (and some of its SNPs) and CCPA (main type of CPA) [8]. They speculated that the continued rising of IL-1 might reflect the inflammatory response to the ongoing fungal infection, which could manifest as tissue damage, cavity formation, and aspergilloma formation. Caffrey et al. also confirmed the role of IL-1 in controlling aspergillus fumigatus infection in the murine lung and speculated the important role of IL-1 in pulmonary fungal infection [24]. However, many of these studies are preclinical and focused on the role of IL- 1 in pathogenesis rather than using it as a biomarker to trace disease activity. In our study, we have included by far the largest number of CPA cases to study the association of IL$1 \mathrm{~B}$ level with the disease activity of CPA. We have therefore added another piece of evidence to support the role of IL-1B in CPA, that is, IL-1B is important not only in the pathogenesis of aspergillosis, but also in the activity of this particular disease.
As per the 2016 new guidelines for CPA, the enlarging lung cavitation and/or progression with more tissue damage and likely poor therapeutic responses [16]. Finding a convenient approach to monitor the disease activity is therefore urgently needed. Since the radiographic appearance and histopathological features are closely related to immune and inflammatory response rather than just direct invasion by fungi $[1,25,26]$, cytokine should theoretically have the role to monitor CPA activity. This is clinically important since the follow-up evaluation of CPA is still insufficient at this moment. The reported clinical, mycological, or radiological criteria are not standardized and vary from study to study. Although the size or number of cavities and fungus ball, as well as the cavity and pleural wall thickness shown in CT scans are commonly used to assess disease status and treatment response, the criteria are not consistent $[17,18,27-29]$. In addition, only little change is visible on CT scans 3 months after the inception of antifungal therapy [16]. Due to the requirement of long follow-up intervals, CPA evaluation by chest CT is not the most effective assessment in 

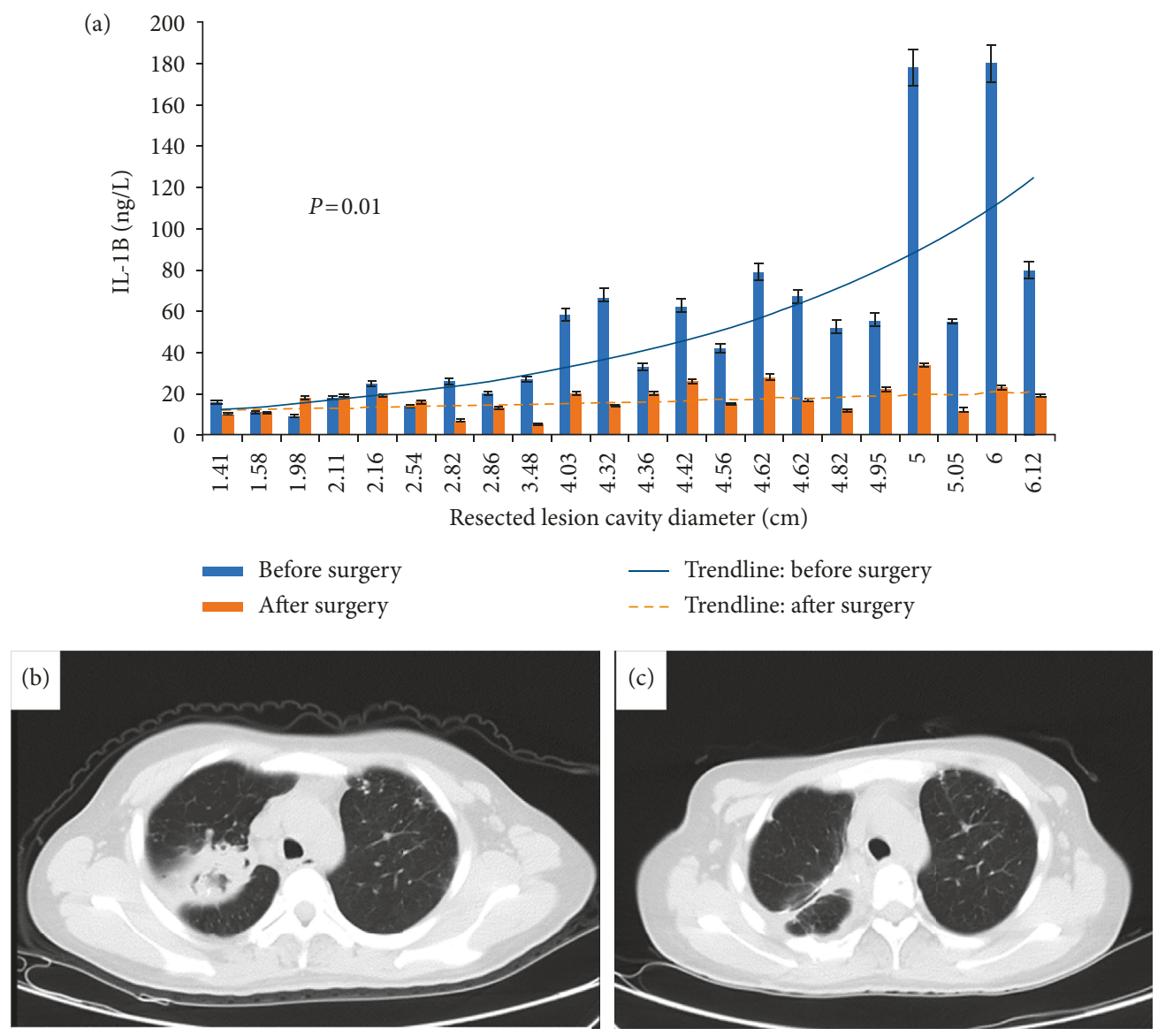

Figure 2: The comparison of IL-1B levels before and after surgery: (a) each dot represents an individual CPA patient. There was a clear upward trend of IL-1B level with the increase of lesion size before the surgery (solid line) and one month later after the surgery; almost all patients' serum IL-1 decreased ( $61.3 \pm 42.7 \mathrm{ng} / \mathrm{L}$ vs $23.7 \pm 9.4 \mathrm{ng} / \mathrm{L}, P=0.001)$ (dotted line), especially in patients whose baseline IL-1 levels were over $20 \mathrm{ng} / \mathrm{L}$. (b) The CT scan of a 63-year-old male CPA patient with multiple cavities, pleural thickening, aspergilloma, and consolidation in the left upper lobe. His serum IL-1 was $180 \mathrm{ng} / \mathrm{L}$ prior to the surgery. (c) 3 months later after he received a left upper lobectomy, no CPA lesions were seen in his chest CT and his serum IL-1 decreased to $23 \mathrm{ng} / \mathrm{L}$.

our clinical work. Other tests such as serum IgG level [30] and GM test [31], etc. have also been considered attractive in monitoring pulmonary fungal infection. However, they are not as good as IL-1B in our study. Since the serum level of IL1B showed good correlation with CT findings that are indicative of disease activity, it might also be useful to predict therapeutic response for CPA. Indeed, among 22 patients who got their cavitary lesions resected, the IL-1B level was found significantly reduced. We will address whether similar trend can be observed among patients who respond well to antifungal therapy, and how fast the change of IL-1B level will be after treatment in future prospective studies.

Several important issues and limitations should be mentioned here. First, this is a retrospective study conducted only in our hospital; therefore, selection bias is inevitable. Second, our whole group of recruited subjects with CPA is not a homogenous population and it includes patients with a variety of infectious and noninfectious comorbidities and some patients taking systemic CS or IS therapy, which is supposed to affect IL-1B expression. For this problem, our group analyzed the association between IL-1B and disease activity first as a whole and then in a subgroup population without confounding factors affecting IL-1B expression, which were identified by performing a multiple linear regression. And at both levels, our results convincingly support a role for IL-1B in the activity of CPA, especially in the latter. In addition, though IL-1 family contains large numbers of members related to Aspergillus fumigatus [32], we only measured IL-1B due to the limitation of the detection kit, which may lead to the missing of some important findings such as factors relating to IL-1B regulator or receptor.

\section{Conclusions}

Nevertheless, our study has shown the association of serum IL-1B level with the disease activity of CPA. The higher level of serum IL-1B is associated with more and bigger cavitary and aspergilloma lesions, which are indicative of more active CPA. In addition, IL-1B level reduced accordingly after 


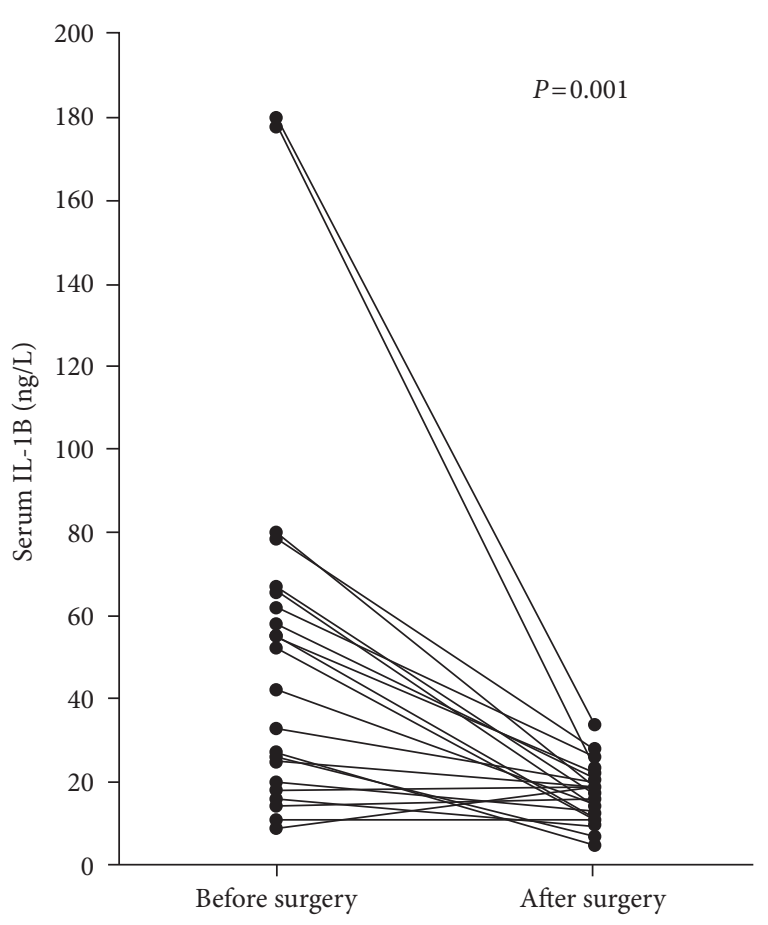

FIgURE 3: The comparison of serum IL-1B concentrations before and after surgery.

lesion resection. Measuring IL-1B level therefore could serve as a convenient way to monitor the activity of CPA and be a potential predictive/prognostic marker for treatment response.

\section{Data Availability}

The data used to support the findings of this study are included within the article.

\section{Conflicts of Interest}

The authors have no conflicts of interest to declare.

\section{Authors' Contributions}

Mengling Zhan and Benyong Xu contributed equally to this work.

\section{Acknowledgments}

We are sincerely thankful to Professor Shengxiang Ren for his careful reviewing and editing of the manuscript, as well as many useful suggestions and assistances. Dr. Haifeng Wang helped writing and editing this manuscript. This project was supported by grants obtained from the National Natural Science Foundation of China (nos. 81672063 and 81800003), Natural Science Foundation of Shanghai Municipal Science and Technology Commission (no. 18ZR1431600), Medical Guide Program of Shanghai Science and Technology Committee (no. 14411962900), Key project of Shanghai Municipal Health and Family Planning Commission (no. 201540367), Youth Project of Shanghai
Municipal Health and Family Planning Commission (no. 20164Y0230), New Frontier Technology Joint Project of Municipal Hospital, Shanghai Shenkang Hospital Development Center (no. SHDC12017113), and Project of Top Clinical Medicine Centers and Key Disciplines Construction in Shanghai (no. 2017ZZ02012).

\section{References}

[1] J. R. Davidsen, F. S. Rosenvinge, K. Assing, and C. B. Laursen, "Chronic pulmonary aspergillosis," Ugeskrift for Laeger, vol. 180, no. 9, pp. 2-6, 2018.

[2] D. W. Denning and D. S. Perlin, "Azole resistance in Aspergillus: a growing public health menace," Future Microbiology, vol. 6, no. 11, pp. 1229-1232, 2011.

[3] D. W. Denning, A. Pleuvry, and D. C. Cole, "Global burden of allergic bronchopulmonary aspergillosis with asthma and its complication chronic pulmonary aspergillosis in adults," Medical Mycology, vol. 51, no. 4, pp. 361-370, 2013.

[4] C. Kosmidis and E. G. Muldoon, "Challenges in the management of chronic pulmonary aspergillosis," Medical Mycology, vol. 55, no. 1, pp. 63-68, 2017.

[5] K. Koyama, N. Ohshima, J. Suzuki et al., "Evaluation of clinical characteristics and prognosis of chronic pulmonary aspergillosis depending on the underlying lung diseases: emphysema vs prior tuberculosis," Journal of Infection and Chemotherapy, vol. 21, no. 11, pp. 795-801, 2015.

[6] H. Ohba, S. Miwa, M. Shirai et al., "Clinical characteristics and prognosis of chronic pulmonary aspergillosis," Respiratory Medicine, vol. 106, no. 5, pp. 724-729, 2012.

[7] B. W. Jhun, K. Jeon, J. S. Eom et al., "Clinical characteristics and treatment outcomes of chronic pulmonary aspergillosis," Medical Mycology, vol. 51, no. 8, pp. 811-817, 2013.

[8] N. L. Smith, J. Hankinson, A. Simpson et al., "A prominent role for the IL1 pathway and IL15 in susceptibility to chronic cavitary pulmonary aspergillosis," Clinical Microbiology and Infection, vol. 20, no. 8, pp. O480-O488, 2014.

[9] N. L. Smith and D. W. Denning, "Clinical implications of interferon-gamma genetic and epigenetic variants," Immunology, vol. 143, no. 4, pp. 499-511, 2014.

[10] B. J. Murdock, A. B. Shreiner, R. A. McDonald et al., "Coevolution of $\mathrm{TH} 1, \mathrm{TH} 2$, and $\mathrm{TH} 17$ responses during repeated pulmonary exposure to Aspergillus fumigatus conidia," Infection and Immunity, vol. 79, no. 1, pp. 125-135, 2011.

[11] E. K. Rodland, T. Ueland, S. Bjornsen et al., "Systemic biomarkers of inflammation and haemostasis in patients with chronic necrotizing pulmonary aspergillosis," BMC Infectious Diseases, vol. 12, no. 1, p. 144, 2012.

[12] C. A. Dinarello, "Overview of the IL-1 family in innate inflammation and acquired immunity," Immunological Reviews, vol. 281, no. 1, pp. 8-27, 2018.

[13] J. Loeffler, Z. Haddad, M. Bonin et al., "Interaction analyses of human monocytes co-cultured with different forms of Aspergillus fumigatus," Journal of Medical Microbiology, vol. 58, no. 1, pp. 49-58, 2009.

[14] N. Said-Sadier, E. Padilla, G. Langsley, and D. M. Ojcius, "Aspergillus fumigatus stimulates the NLRP3 inflammasome through a pathway requiring ROS production and the Syk tyrosine kinase," PLoS One, vol. 5, no. 4, Article ID e10008, 2010.

[15] H. Sun, X. Y. Xu, X. L. Tian et al., "Activation of NF-kappaB and respiratory burst following Aspergillus fumigatus stimulation of macrophages," Immunobiology, vol. 219, no. 1, pp. 25-36, 2014. 
[16] D. W. Denning, J. Cadranel, C. Beigelman-Aubry et al., "Chronic pulmonary aspergillosis: rationale and clinical guidelines for diagnosis and management," European Respiratory Journal, vol. 47, no. 1, pp. 45-68, 2016.

[17] R. Agarwal, G. Vishwanath, A. N. Aggarwal, M. Garg, D. Gupta, and A. Chakrabarti, "Itraconazole in chronic cavitary pulmonary aspergillosis: a randomised controlled trial and systematic review of literature," Mycoses, vol. 56, no. 5, pp. 559-570, 2013.

[18] C. Godet, F. Laurent, A. Bergeron et al., "CT Imaging assessment of response to treatment in chronic pulmonary Aspergillosis," Chest, vol. 150, no. 1, pp. 139-147, 2016.

[19] S. R. Desai, V. Hedayati, K. Patel, and D. M. Hansell, "Chronic Aspergillosis of the lungs: unravelling the terminology and radiology," European Radiology, vol. 25, no. 10, pp. 31003107, 2015.

[20] D. J. Crosdale, K. V. Poulton, W. E. Ollier, W. Thomson, and D. W. Denning, "Mannose-binding lectin gene polymorphisms as a susceptibility factor for chronic necrotizing pulmonary aspergillosis," Journal of Infectious Diseases, vol. 184, no. 5, pp. 653-656, 2001.

[21] M. Vaid, S. Kaur, H. Sambatakou, T. Madan, D. W. Denning, and P. Usha Sarma, "Distinct alleles of mannose-binding lectin (MBL) and surfactant proteins A (SP-A) in patients with chronic cavitary pulmonary aspergillosis and allergic bronchopulmonary aspergillosis," Clinical Chemical Laboratory Medicine, vol. 45, no. 2, pp. 183-186, 2007.

[22] H. Sambatakou, V. Pravica, I. V. Hutchinson, and D. W. Denning, "Cytokine profiling of pulmonary aspergillosis," International Journal of Immunogenetics, vol. 33, no. 4, pp. 297-302, 2006.

[23] A. Carvalho, A. C. Pasqualotto, L. Pitzurra, L. Romani, D. W. Denning, and F. Rodrigues, "Polymorphisms in tolllike receptor genes and susceptibility to pulmonary aspergillosis," Journal of Infectious Diseases, vol. 197, no. 4, pp. 618-621, 2008.

[24] A. K. Caffrey, M. M. Lehmann, J. M. Zickovich et al., "ILlalpha signaling is critical for leukocyte recruitment after pulmonary Aspergillus fumigatus challenge," PLoS Pathogens, vol. 11, no. 1, article e1004625, 2015.

[25] G. E. Hayes and L. Novak-Frazer, "Chronic pulmonary Aspergillosis-where are we? and where are we going?", Journal of Fungi, vol. 2, no. 2, p. 18, 2016.

[26] A. H. Henderson, "Allergic aspergillosis: review of 32 cases," Thorax, vol. 23, no. 5, pp. 501-512, 1968.

[27] D. W. Denning, K. Riniotis, R. Dobrashian, and H. Sambatakou, "Chronic cavitary and fibrosing pulmonary and pleural aspergillosis: case series, proposed nomenclature change, and review," Clinical Infectious Diseases, vol. 37, no. S3, pp. S265-S280, 2003.

[28] T. W. Felton, C. Baxter, C. B. Moore, S. A. Roberts, W. W. Hope, and D. W. Denning, "Efficacy and safety of posaconazole for chronic pulmonary aspergillosis," Clinical Infectious Diseases, vol. 51, no. 12, pp. 1383-1391, 2010.

[29] J. Cadranel, B. Philippe, C. Hennequin et al., "Voriconazole for chronic pulmonary aspergillosis: a prospective multicenter trial," European Journal of Clinical Microbiology \& Infectious Diseases, vol. 31, no. 11, pp. 3231-3239, 2012.

[30] Y. Yao, H. Zhou, Q. Yang et al., "Serum Aspergillus fumigatus-specific IgG antibody decreases after antifungal treatment in chronic pulmonary aspergillosis patients," Clinical Respiratory Journal, vol. 12, no. 4, pp. 1772-1774, 2018.
[31] S. Eigl, J. Prattes, M. Reinwald et al., "Influence of mouldactive antifungal treatment on the performance of the Aspergillus-specific bronchoalveolar lavage fluid lateral-flow device test," International Journal of Antimicrobial Agents, vol. 46, no. 4, pp. 401-405, 2015.

[32] M. S. Gresnigt and F. L. Van De Veerdonk, "The role of interleukin-1 family members in the host defence against Aspergillus fumigatus," Mycopathologia, vol. 178, no. 5-6, pp. 395-401, 2014. 


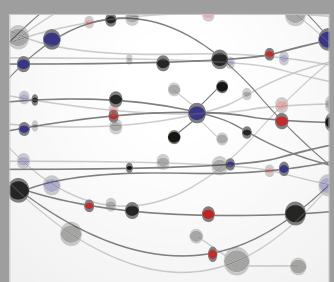

The Scientific World Journal
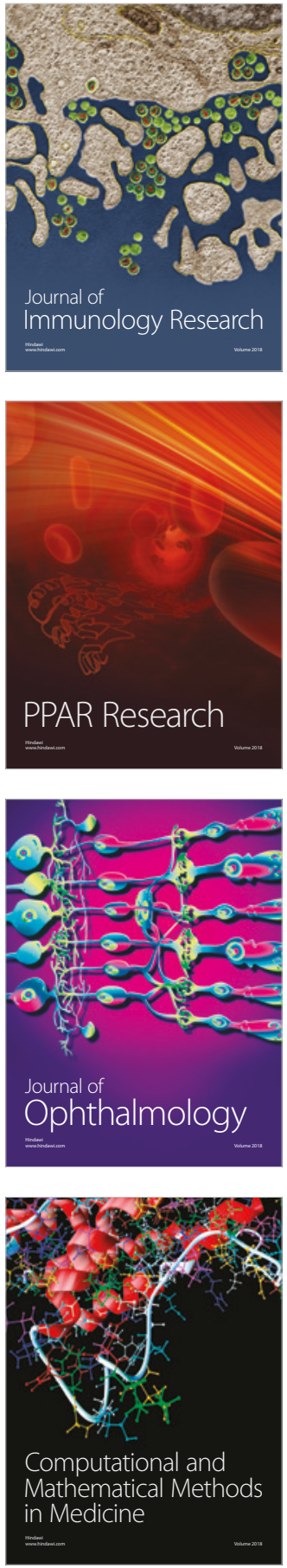

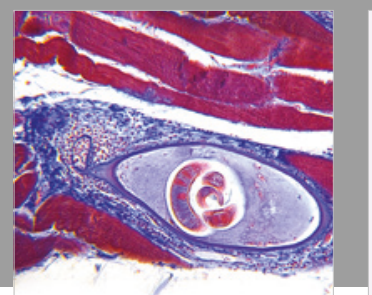

Gastroenterology Research and Practice

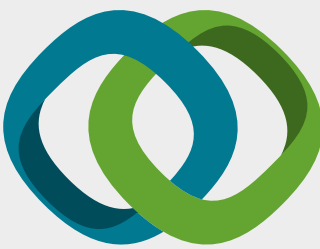

\section{Hindawi}

Submit your manuscripts at

www.hindawi.com
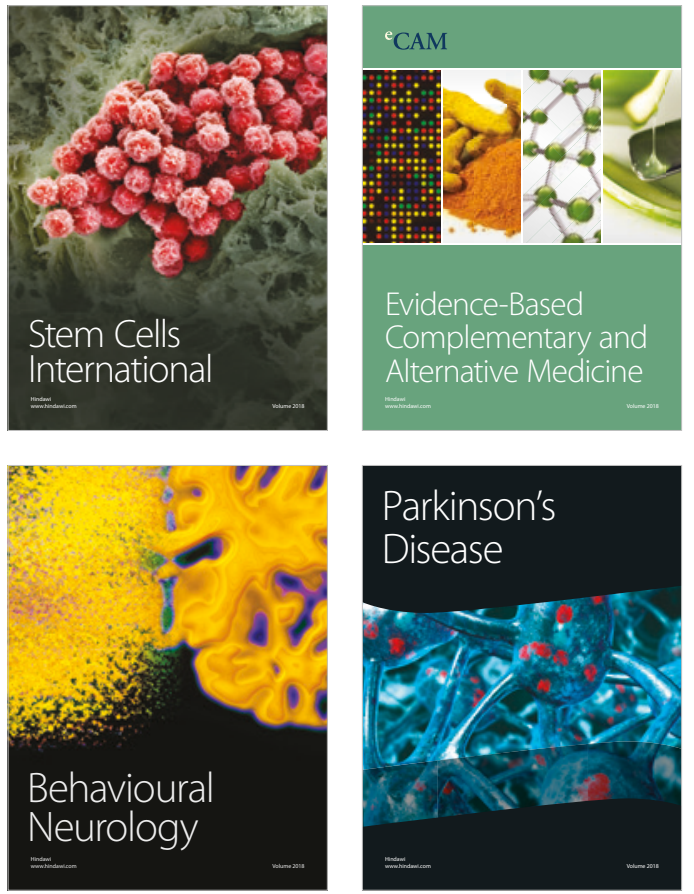

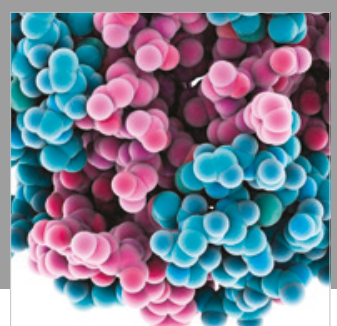

ournal of

Diabetes Research

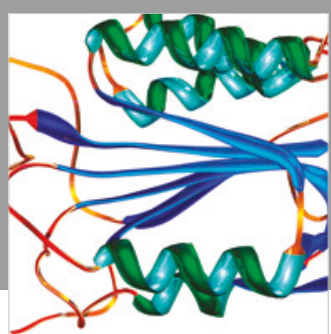

Disease Markers
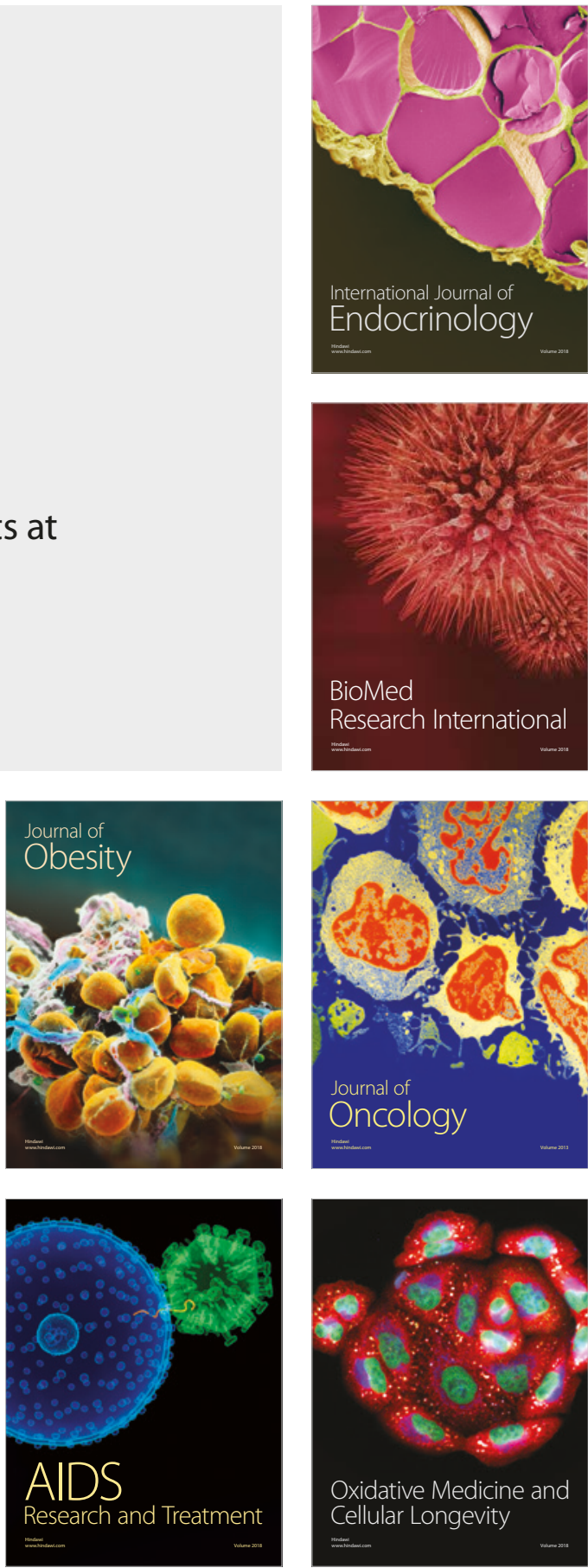